\title{
ON THE LOGARITHM OF A UNIFORMLY BOUNDED OPERATOR $\left({ }^{1}\right)$
}

\author{
BY \\ G. L. KRABBE
}

1. Introduction. Let $Y$ be a bounded operator on a complex Banach space, and denote by $\mathscr{R}(Y)$ the set of all bounded operators $B$ such that $\exp B=Y$. If $\&$ is an arbitrary subset of the plane, we shall say that $N_{8}(Y)=1$ if there exists a unique member $A$ of $\mathfrak{R}(Y)$ satisfying the following two conditions:

(i) The spectrum of $A$ is included in the closure of 8 .

(ii) Any bounded operator which commutes with $Y$ commutes then also with $A$.

Suppose $N_{8}(Y)=1$; we can then define $\log _{8}(Y)$ as the unique such $A$. It is readily seen (Theorem $I V, \S 3$ ) that any $B$ in $\mathscr{R}(Y)$ determines $\left({ }^{2}\right)$ a member $Q$ of $\mathcal{L}(I)$ such that $B=\log _{8}(Y)+Q$. Of special interest is the case $8=e$ $=\{\lambda|| \operatorname{Im} \lambda \mid<\pi\}$ corresponding to the principal value of the logarithm.

If 0 is in the principal component $\rho_{1}(Y)$ of the resolvent set of $Y$, there exist sets such that $N_{8}(Y)=1$; this is shown in $\$ 3$ to be a consequence of the Dunford operational calculus. Conversely, this calculus is inapplicable [2, p. 125] if $0 \notin \rho_{1}(Y)$; in fact, it is beyond its power to exhibit one single member of $\mathfrak{R}(Y)$ for such operators $Y$. In 1.1 we give an example of an operator $Y$ such that $0 \notin \rho_{1}(Y)$ and $\mathfrak{R}(Y) \neq 0$. The condition $0 \in \rho_{1}(Y)$ is therefore not necessary $\left(^{3}\right)$ for $\mathfrak{L}(Y) \neq 0$, and will be omitted, in certain cases considered here.

In particular, if $Y$ is a unitary operator on a Hilbert space, then $N_{\mathrm{e}}(Y)=1$ when neither 1 nor -1 are eigenvalues of $Y$ (see $\$ 9)$. Attempts at extending this property lead naturally to the class $\mathfrak{U}$ of all operators $Y$ defined on a reflexive space and satisfying sup $\left\{\left\|Y^{n}\right\| \mid n=0, \pm 1, \pm 2, \pm 3, \cdots\right\}<\infty$. Members of $\mathfrak{U}$ were called "uniformly bounded transformations" by $E$. $R$. Lorch $\left({ }^{4}\right)$, who first studied their properties.

Presented to the Society, September 2, 1954; received by the editors October 26, 1954.

(1) The main results of this paper are contained in the author's doctoral dissertation at the University of California. The author wishes to express his gratitude to Professor C. B. Morrey, Jr., for his guidance. $\$ 3$.

(2) The operator $I$ is the identity; the elements of $\mathscr{R}(I)$ are characterized in Theorem III,

(3) See [2, p. 452]. Another example is given by Lorch [5], who treated related questions in the commutative case; for instance, his Theorem 10 corresponds to our Theorem III.

(4) See $[3 ; 4]$. Sz.-Nagy [10] has shown that, in the special case where a member $Y$ of $\mathfrak{U}$ is defined on a Hilbert space, then $Y=H U H^{-1}$ for some operators $H$ and $U$, respectively selfadjoint and unitary. 
Suppose that $Y \in \mathfrak{U}$, and that neither 1 nor -1 are eigenvalues of $Y$. Our main result (Theorem VI) states that there exists at most one bounded operator $A$ with $\exp (i A)=Y$ whose spectrum lies on the closed interval $[-\pi, \pi]$; moreover, $N_{\mathrm{e}}(Y)=1$ if and only if such an $A$ exists. Our proofs depend largely on the spectral resolution constructed by Lorch for such operators [3], which enables us, in a sense, to "split" (in \$7) the spectrum of $Y$.

A fundamental role is performed here by the relation defined by (ii) for arbitrary bounded operators $Y$ and $A$. It follows from Theorem I that this relation holds whenever $A$ is a function of $Y$ (in the sense of Dunford's operational calculus), provided the spectrum of $Y$ does not separate the plane. We note that $\mathfrak{U}$ and $\mathfrak{A}=\{i A \mid A \in \mathfrak{R}(Y), Y \in \mathfrak{U}\}$ include the class of unitary and bounded self-adjoint operators, respectively. If $X$ belongs to either $\mathfrak{U}$ or $\mathfrak{A}$, then all isolated points of the spectrum of $X$ are eigenvalues of $X$. Moreover, the residual spectrum of $X$ is empty when $X \in \mathfrak{A}$, or in case $X \in \mathfrak{U}$ and $\mathfrak{R}(X) \neq 0$ (Theorem $\mathrm{V}, 6.5$ ).

1.1. Application. Let $\langle a\rangle$ denote the infinite matrix $\left(a_{n-v}\right),-\infty<n$, $\nu<+\infty$. Let $b_{n}=0$ if $n \neq-1, b_{-1}=1$, and $c_{n}=i(-1)^{n} / n, c_{0}=0$. The matrices $\langle b\rangle$ and $\langle c\rangle$ represent two transformations $E_{*}^{1}$ and $I_{*}$ on any one of the reflexive spaces $l_{p}, p>1$. It has been established $\left(^{(5)}\right.$ that these are bounded operators whose spectra are, respectively, the whole circumference of the unitcircle, and the interval $[-\pi, \pi]$; moreover $\exp \left(-i I_{*}\right)=E_{*}^{1}$.

We thus have an illustration of the case $0 \notin \rho_{1}\left(E_{*}^{1}\right), \mathfrak{l}\left(E_{*}^{1}\right) \neq 0$. Note that $E_{*}^{1}$ has no eigenvalues( $\left.{ }^{5}\right)$ and $E_{*}^{1} x=\left\{x_{n+1} \mid n\right\}, x \in l_{p}$. Therefore $E_{*}^{1} \in \mathfrak{U}$, and the conclusion $N_{\mathrm{e}}\left(E_{*}^{1}\right)=1,-i I_{*}=\log _{\mathrm{e}}\left(E_{*}^{1}\right)$ now follows from Theorem VI.

\section{Preliminaries.}

2.1. Notation. Linear transformations which map a fixed Banach space into itself will be called operators; these we denote by $A, B, X, Y, Z, I$ (the identity), and 0 (the zero). The values of $\lambda$ for which $\lambda I-Y$ has a bounded inverse operator $\tilde{Y}_{\lambda}$ form the resolvent set $\rho(Y)$ of $Y$. The component of $\rho(Y)$ containing the point at infinity will be symbolized by $\rho_{1}(Y)$.

We shall write $Y \prec B$ if any bounded operator commuting with $Y$ commutes then also with $B$. The following properties are easily verified:

(a) $X \prec Y$ implies $X Y=Y X$,

(b) $X \prec Y$ and $Y \prec A$ implies $X \prec A$,

(c) $\sum_{n=-\infty}^{\infty} \lambda_{n} Y^{n}=X$ implies( $\left.{ }^{6}\right) \quad Y \prec X$.

2.2. Lemma( $\left.{ }^{7}\right)$. Suppose $A \in \mathbb{R}(Y)$ and $Y<A$; then any $B$ in $\mathfrak{R}(Y)$ deter-

(5) See G. L. Krabbe, Abelian rings and spectra of operators on $l_{p}$, to appear in Proc. Amer. Math. Soc.

(6) The $\lambda_{n}$ are complex, and convergence is assumed; see 7.1 .

(7) We recall that $A \in \mathbb{Q}(Y)$ if $\exp A=\sum_{n-0}^{\infty}(n !)^{-1} A^{n}=Y$. This definition of $\exp (A)=f(A)$ agrees with the interpretation of $f(A)$ which will be adopted in 3.3 [1, Theorem 2.8]. 
mines a member $Q$ of $\mathfrak{R}(I)$ such that $B=A+Q$. Moreover $B<Y, B<A$, and $B<Q$.

Proof. We derive $B \prec Y$ from (c) and the hypothesis $\exp B=Y$; but $Y \prec A$, so that $B<A$ and $B A=A B$ follow from (b) and (a) respectively. Therefore $[2$, p. 124]

$$
\exp (B-A)=(\exp B)(\exp -A)=Y Y^{-1}=I .
$$

If we now call $Q=B-A$, then $\exp Q=I$ and $B=A+Q$. The proof is concluded by noting that $B<Q$ follows from $B<A$ and $A=B-Q$.

2.3. Lemma. If $\alpha \in \rho_{1}(Y)$, then $Y \prec \tilde{Y}_{\alpha}$.

Proof. If $\lambda \in \mathcal{E}=\{\lambda|| \lambda \mid>\|Y\|\}$, then [2, p. 98] $\tilde{Y}_{\lambda}=\sum_{n=0}^{\infty} Y^{n} \lambda^{-n-1}$ and we can infer from (c) that $Y \prec \widetilde{Y}_{\lambda}$. This means that if $Z Y=Y Z$, then the functions $Z \cdot \tilde{Y}_{\lambda}$ and $\tilde{Y}_{\lambda} \cdot Z$ coincide on the subregion of the region $\rho_{1}(Y)$; these two functions of $\lambda$ are both analytic of $\lambda$ on $\rho_{1}(Y)$, and the uniqueness theorem $\left[2\right.$, p. 57] now shows that these functions coincide throughout $\rho_{1}(Y)$. This concludes the proof.

3. The logarithm of a bounded operator. The relation $Y<X$ generalizes

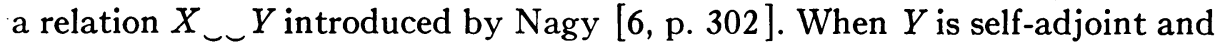
$f(Y)=X$, then $X_{\smile} Y$ holds, provided $f(Y)$ is interpreted in the sense of the functional calculus for such operators [6, p. 341]. We shall prove in 3.3 an analogous result for bounded operators $Y$.

3.1. Notation. The letters $\mathfrak{a}, \mathfrak{b}, \&$ will be reserved for sets in the plane; $\sim \mathfrak{b}$ denotes the complement of $\mathfrak{b}$. Thus $\sigma(Y)=\sim \rho(Y)$ is the spectrum of $Y$.

3.2. REMARK. If $\sigma$ is a bounded and closed set, we shall write $\mathfrak{a} \in \mathcal{C}(\sigma)$ when $\mathfrak{a}$ is a bounded and open set containing $\sigma$, whose boundary $\partial \mathfrak{a}$ consists of a finite family of (suitably oriented) contours in $\sim \sigma$. A simple argument of the Heine-Borel type $[6$, p. $420 ; 7$, p. 193] shows that if $\mathfrak{b}$ is an open set with $\mathfrak{b} \supset \sigma$, then $\mathfrak{b}$ contains the closure of some $\mathfrak{a}$ in $\mathcal{A}(\sigma)$.

3.3. Theorem I. Suppose $f$ is holomorphic $\left({ }^{8}\right)$ on some open set $\mathfrak{b} \supset \sigma_{1}$ $=\sim \rho_{1}(Y)$. If $f(Y)=X$, then $Y \prec X$.

Proof. As in 3.2, we can infer that $\mathfrak{b}$ contains the closure of some $\mathfrak{a}$ in $A\left(\sigma_{1}\right)$. Observe that $a \supset \sigma_{1} \supset \sigma(Y)$, and $\partial \mathfrak{a} \subset \sim \sigma_{1} \subset \rho(Y) ; \mathfrak{a}$ is therefore " $Y$ admissible" [6, p. 420], and consequently $\int_{k} f(\lambda) \widetilde{Y}_{\lambda} d \lambda(k=\partial \mathfrak{a})$ is independent of the choice of $\mathfrak{a}$ (satisfying the above conditions), and defines a bounded operator customarily written $f(Y)$.

In order to show that $Y \prec f(Y)$, we suppose $Z Y=Y Z$ and note that $Z \tilde{Y}_{\lambda}=\tilde{Y}_{\lambda} Z, \lambda \in \rho_{1}(Y)$, follows from 2.3 ; this holds "a fortiori" for $\lambda \in k$ $=\partial \mathfrak{a} \subset \rho_{1}(Y)$, and therefore

$\left.{ }^{8}\right)$ We say that $f$ is holomorphic on $\mathfrak{b}$ whenever $f$ is a one-valued function, defined and analytic on $\mathfrak{b}$. 


$$
Z \cdot f(Y)=\frac{1}{2 \pi i} \int_{k} f(\lambda) Z \cdot \tilde{Y}_{\lambda} d \lambda=\frac{1}{2 \pi i} \int_{k} f(\lambda) \tilde{Y}_{\lambda} \cdot Z d \lambda=f(Y) \cdot Z .
$$

This concludes the proof.

3.4. Definitions. We say with Saks $[8, \mathrm{pp} .74-75]$ that $f$ is a branch of the logarithm in $\mathfrak{b}$ if $f$ is holomorphic in $\mathfrak{b}$, if $f \neq \infty$ in $\mathfrak{b}$, and $\exp (f(\alpha))=\alpha$ when $\alpha \in \mathfrak{b}\left({ }^{9}\right)$. Let $\mathbb{B}(Y)$ be the family of all open sets $\mathfrak{b}$ with $\{0, \infty\} \subset \sim \mathfrak{b}$, and such that $\sim \mathfrak{b}$ is a connected subset of $\rho_{1}(Y)$.

3.5. ThEOREM. If $\mathfrak{b} \in \mathbb{B}(Y)$, there exists a branch $f$ of the logarithm in $\mathfrak{b}$. Moreover, $f$ and $b$ are related as in 3.3, and $A=f(Y)$ is the only member of $\mathfrak{L}(Y)$ such that $\sigma(A) \subset f(\mathfrak{b})$.

Proof. The existence of $f$ follows from [8, p. 180]. Since $f$ is holomorphic on $\mathfrak{b} \supset \sim \rho_{1}(Y) \supset \sigma(Y)$, we can deduce from the Dunford mapping theorem $\left({ }^{10}\right)$ that $\sigma(f(Y))=f(\sigma(Y)) \subset f(\mathfrak{b})$; the conclusion $\sigma(A) \subset f(\mathfrak{b})$ now follows from $A=f(Y)$. The property $\exp A=Y$ is a consequence of the relation $\exp (f(\alpha))$ $=\alpha$ holding for $\alpha \in \mathfrak{b} \supset \sigma(Y)$, and a certain theorem by Dunford( $\left.{ }^{11}\right)$ which yields $\exp (f(Y))=Y$. Suppose $B \in \mathfrak{R}(Y), \sigma(B) \subset f(\mathfrak{b})$; it remains only to show that $B=A$. To that effect, note $\left.{ }^{9}\right)$ that $f(\exp \lambda)=\lambda$ for $\lambda \in f(\mathfrak{b}) \supset \sigma(B)$ and thus $\left.{ }^{11}\right) f(\exp B)=B$. Consequently $f(Y)=B$, and $B=A$.

3.6. REMARK. It is easily checked that

$$
\mathbb{B}(Y) \neq 0 \text { if and only if } 0 \in \rho_{1}(Y) \text {. }
$$

The hypothesis $\mathbb{B}(Y) \neq 0$ of 3.5 is therefore $[2, \mathrm{p} .125]$ the most general condition on $Y$ which is compatible with the Dunford operational calculus.

3.7. TheOREM II. For any set \& such that $\exp (\mathbb{B}) \in \mathcal{B}(Y)$, there exists a unique $A$ in $\mathfrak{Q}(Y)$ satisfying $\sigma(A) \subset$. Moreover $Y<A$ for such an $A$.

This is an immediate consequence of 3.5 and 3.3 , in view of the fact that $\left({ }^{9}\right)$ $f(\exp 8)=8$.

3.8. Remark. Suppose $\alpha$ is a real number. If $\xi_{\alpha}$ denotes the horizontal strip $\{\lambda|| \operatorname{Im} \lambda-\alpha \mid<\pi\}$, then $\sim \exp \left(B_{\alpha}\right)$ is the half-line $\mathfrak{I}_{\alpha}=\left\{p e^{i \alpha} \mid p \leqq 0\right\}$. The following corollary of 3.7 will be needed: for any $\mathfrak{l}_{\alpha} \subset \rho(Y)$ there exists a unique member $A$ of $\mathfrak{R}(Y)$ such that $\sigma(A) \subset \xi_{\alpha}$; furthermore (in view of 3.5), $A=f(Y)$, where $f$ is one-to-one $\left({ }^{9}\right)$ and holomorphic on $\mathfrak{b}=\sim \mathfrak{l}_{\alpha} \supset \sigma(Y)$. In the particular case $\mathfrak{l}_{0}=(-\infty, 0]$, call $\xi_{0}=\mathrm{e}$; $\operatorname{then}\left({ }^{12}\right) N_{\mathrm{e}}(Y)=1$, provided $\sigma(Y)$ is included in the cut-plane $\sim(-\infty, 0]$. Note that $\log _{e}(Y)=f(Y)$, where $f$ is now the principal value of the logarithm.

( $\left.{ }^{9}\right)$ Therefore $f$ is one-to-one on $\mathfrak{b}$, and $f(\exp \lambda)=\lambda$ for $\lambda$ in $f(\mathfrak{b})$.

(10) See 6.2 , also $[6$, p. $421,(28)]$.

(11) See $[6$, p. 422$]$, also $[1$, p. 196, 2.10].

(12) The notation used here is defined in the introduction. 
3.9. THEOREM III. The operator $Q$ is in $\mathfrak{R}(I)$ if and only if there exists a finite family $\left\{P_{n}\right\}$ of operators satisfying

$$
Q=2 \pi i \sum_{n} n P_{n}, \quad \sum_{n} P_{n}=I, \quad \text { and } P_{n} P_{\nu}=0 \text { for } n \neq \nu .
$$

Proof. Since $P_{n}=\left(P_{n}\right)^{2}$, it is quickly verified that the condition is sufficient [2, Theorem 5.18.2]. On the other hand, if $\exp Q=I$, then $\left({ }^{10}\right) \exp (\sigma(Q))$ $=\sigma(\exp Q)=\sigma(I)=\{1\}$; hence $\sigma(Q) \subset\{2 \pi i n \mid n=0, \pm 1, \pm 2, \pm 3, \cdots\}$. To every $n$ corresponds $\left[2\right.$, p. 105] an operator $P_{n}$ with $\sum_{n} P_{n}=I$ and $P_{n} P_{\nu}=0$ for $n \neq \nu$. If $Q_{n}$ and $I_{n}$ denote the restriction of $Q$ and $I$, respectively, to the range of $P_{n}$, then (see 7.2) $Q_{n} \in \mathfrak{R}\left(I_{n}\right)$; moreover $\left[6\right.$, p. 410] $\sigma\left(Q_{n}\right) \subset\{2 \pi n i\} \subset \varepsilon_{\alpha}$ $(\alpha=2 \pi n)$. But the operator $2 \pi n i I_{n}$ also has these properties; we therefore deduce from 3.8 that $Q_{n}=2 \pi n i I_{n}$. The conclusion follows from $Q=Q \sum_{n} P_{n}$ $=\sum_{n} Q_{n} P_{n}=\sum_{n} 2 \pi n i P_{n}$.

3.10. Theorem IV. If $N_{8}(Y)=1$, then $B=\log _{8}(Y)+Q$ for any $B$ in $\mathbb{R}(Y)$ and some $Q$ in $\mathbb{R}(I)$. Moreover $B<Y<\log _{8}(Y)<Y$ and $B<Q$. The hypothesis $N_{8}(Y)=1$ is satisfied for some set 8 , in case $0 \in \rho_{1}(Y)$; we then have $\log _{8}(Y)$ $=f(Y)$, where $f$ is a branch of the logarithm in $\exp (8)$.

Proof. This is an obvious consequence of 2.2, 3.6, 3.7, and 3.5. (The symbolism was defined in the introduction.)

3.11. Orientation. We shall henceforth investigate operators $Y$ such that $\sigma(Y) \subset \Gamma_{0}=\{\lambda|| \lambda \mid=1\}$; the Dunford functional calculus is not applicable when $\sigma(Y)=\Gamma_{0}$ (since $0 \notin \rho_{1}(Y)$ ). Our aim will be to establish $N_{e}(Y)=1$ under conditions which allow the possibility $\sigma(Y)=\Gamma_{0}$.

4. Some results of Lorch.

4.1. Notation. Let $\mathcal{D}(Y)$ denote the domain of $Y$. We shall write $Y \in \mathfrak{U}$ if $\mathcal{D}(Y)$ is a reflexive Banach space, and if moreover $\mathcal{D}(Y)=\mathscr{D}\left(Y^{-1}\right)$ and sup $\left\{\left\|Y^{n}\right\| \mid n=0, \pm 1, \pm 2, \pm 3, \cdots\right\}<\infty$. Let $p(Y)$ denote the pointspectrum of $Y$. The set $\{x \mid x \in \mathcal{D}(Y), Y x=0\}$ will be called the null-space of $Y$.

4.2. Lemma. Suppose $Y \in \mathfrak{u}$. Let $\sum_{n=-\infty}^{\infty} \lambda_{n}^{\prime} Y^{n}$ and $\sum_{n=-\infty}^{\infty} \lambda_{n}^{\prime \prime} Y^{n}$ denote the series (6) and (7) in [3, p. 24]; these series converge (see 7.1) and define two bounded operators $[Y]^{1}$ and $[Y]^{2}$, respectively. Furthermore

$$
\begin{aligned}
{[Y]^{1}[Y]^{2} } & =0=[Y]^{2}[Y]^{1}, \\
{[Y]^{1}+[Y]^{2} } & =(2 i)^{-1}\left(Y-Y^{-1}\right) .
\end{aligned}
$$

These results are found in [3].

4.3. THEOREM. If $Y \in \mathfrak{U}$, then to every real $\lambda$ corresponds a pair of subspaces $\{Y\}_{\lambda}^{1}$ and $\{Y\}_{\lambda}^{2}$ of $\mathcal{D}(Y)$ satisfying:

(a) $\{Y\}_{\mu}^{2} \supset\{Y\}_{\lambda}^{2}$ and $\{Y\}_{\mu}^{1} \subset\{Y\}_{\lambda}^{1}$ if $\mu \leqq \lambda$.

(b) $\{Y\}_{-\pi}^{2}=\mathscr{D}(Y)$ and $\{Y\}_{\pi}^{2}=\{0\}$. 
(c) $\{Y\}_{\lambda}^{\nu}=\mathcal{D}(Y) \leftrightarrows\{Y\}_{\lambda}^{\kappa}=\{0\}$ (when $\left.\nu \neq \kappa, \nu=1,2\right)$.

(d) If $1 \notin p(Y)$ and $-1 \notin p(Y)$, then $\{Y\}_{0}^{\kappa}$ is the null-space of $[Y]^{\star}$.

The statements (a) and (b) are found in [3, Theorem 7]; the notation used there is $\mathfrak{E}_{\lambda}=\{Y\}_{\lambda}^{1}$ and $\mathfrak{F}_{\lambda}=\{Y\}_{\lambda}^{2}$. The proposition (c) follows immediately from [loc. cit.]. To prove (d) we note [3, pp. 30, 29, and 28]

$$
\begin{array}{ll}
\mathfrak{F}_{0}=\mathfrak{M}_{0}=\left\{x \mid x \in \mathcal{D}(Y), A_{0} x=0, g_{0}^{\prime}=0\right\}, & A_{\lambda}=\left[e^{-i \lambda} Y\right]^{1}, \\
\mathfrak{F}_{0}=\mathfrak{N}_{0}=\left\{x \mid x \in \mathcal{D}(Y), B_{0} x=0, g_{0}=0\right\}, & B_{\lambda}=\left[e^{-i \lambda} Y\right]^{2} .
\end{array}
$$

Furthermore, for any $x$ in $\mathcal{D}(Y) ; Y g_{0}=g_{0}$ and $Y g_{0}^{\prime}=-g_{0}^{\prime} \quad[3$, p. 28]. Hence $g_{0}=0$ and $g_{0}^{\prime}=0$, since otherwise either $1 \in p(Y)$ or $-1 \in p(Y)$. The conclusion follows.

4.4. Lemma. If $Y \in \mathfrak{U}$ and $\alpha \in(0, \pi)$, then

$$
\bigcap_{\lambda>\alpha}\{Y\}_{\lambda}^{1} \text { is a subset of }\{Y\}_{\alpha}^{1} \text {. }
$$

Proof. Suppose that $\lambda \in(\alpha, \pi)$; now $\{Y\}_{\lambda}^{1}=\mathbb{E}_{\lambda}$ in Lorch's notation, and $[3$, p. 30]

$$
x \in\{Y\}_{\lambda}^{1} \leftrightarrows A B_{\lambda-\pi} x=-A A_{\lambda} x=0, \quad g_{0}^{\prime}=0 \quad \text { and } \quad x \in \mathscr{D}(Y) .
$$

From (3), (4), and 4.2 we see that $A_{\lambda}$ and $B_{\lambda-\pi}$ are defined by uniformly convergent series, and both are therefore continuous functions of $\lambda$. The proof will be completed when we show that (5) holds for $\lambda=\alpha$ if it holds for all $\lambda$ in $(\alpha, \pi)$. This, however, follows from the continuity of $A_{\lambda}$ and $B_{\lambda-\pi}$ (that $g_{0}^{\prime}$ is independent of $\lambda$ is seen by referring to the definition $[3$, p. 28] of that symbol).

4.5 Remark. Lemma 4.4 shows that $\{Y\}_{\alpha+0}^{1}=\{Y\}_{\alpha}^{1}$, where $\{Y\}_{\alpha+0}^{1}$ is the set-theoretic limit of the monotonic sequence $\{Y\}_{\lambda}^{1}$. The proof of 4.4 can be elaborated to establish the right-continuity of $\{Y\}_{\lambda}^{1}$, from which the corresponding property of $\{Y\}_{\lambda}^{2}$ follows in some cases.

\section{Lorch's spectral analysis in $\mathfrak{U}$.}

5.1. Definitions. Lorch calls $\lambda$ a point of constancy if there exists numbers $p$ and $q$ such that $\lambda \in[p, q]$ and $\{Y\}_{p}^{\alpha}=\{Y\}_{q}^{\alpha}$ for $\kappa=1$, 2. Let us call $R(Y)$ the totality of all points of constancy, and define

$$
\{Y\}_{\alpha-0}^{2}=\bigcap_{\lambda<\alpha}\{Y\}_{\lambda}^{2}
$$

5.2. Lemma. If $Y \in \mathfrak{U}, \alpha \in(0, \pi)$ and $(\alpha, \pi] \subset R(Y)$, then $\{Y\}_{\alpha}^{2}=\{0\}$ $=\{Y\}_{\pi}^{2}$.

Proof. From 4.3(a) and our hypothesis that every point on $(\alpha, \pi]$ is a point of constancy, follows easily that $\{Y\}_{\lambda}^{2}$ must be the same for all $\lambda$ on 
$(\alpha, \pi]$; in fact $\{Y\}_{\lambda}^{2}=\{0\}=\{Y\}_{\pi}^{2}$, and we can infer from 4.3(c) that $\{Y\}_{\lambda}^{1}$ $=\mathscr{D}(Y)$ for $\lambda \in(\alpha, \pi]$. Applying now 4.4 and 4.3(c) in succession, we obtain $\{Y\}_{\alpha}^{1}=\mathcal{D}(Y)$ and $\{Y\}_{\alpha}^{2}=\{0\}$.

5.3. Theorem. Suppose $Y \in \mathfrak{U}$. The residual spectrum of $Y$ is void, and $\sigma(Y) \subset \Gamma_{0}=\{\zeta|| \zeta \mid=1\}$. Moreover, if $|\lambda|<\pi$, then

$$
\begin{aligned}
& \phi(\lambda) \notin \sigma(Y) \leftrightarrows \lambda \in \Re(Y) \\
& \phi(\lambda) \in p(Y) \leftrightarrows\{Y\}_{\lambda-0}^{2} \neq\{Y\}_{\lambda}^{2} .
\end{aligned} \quad\left(\phi(\lambda)=e^{i \lambda}\right),
$$

These results form part of Lorch's Theorem $9[3$, p. 36]; as in the preceding section, we have extracted from the context the only facts needed in this paper.

6. Punctuality.

6.1. Definition. Let $r(X)$ and $\sigma_{0}(X)$ denote, respectively, the residual spectrum of $X$, and the set of isolated points of $\sigma(X)$; we say that $X$ is punctual if $\sigma_{0}(X) \subset p(X)$.

6.2. LemMA. If $f$ is holomorphic on some open set $\mathfrak{b} \supset \sigma(X)$, then $f(\Lambda(X))$ $=\Lambda(f(X))$, where $\Lambda=\sigma, p$. In particular, if $f$ is one-to-one on $\mathfrak{b}$, and $\alpha \in \mathfrak{b}$, then

$$
\alpha \in \Lambda(X) \leftrightarrows f(\alpha) \in \Lambda(f(X)) \quad(\Lambda=\sigma, p, r) .
$$

The proof is given in 9.6.

6.3. Lemma. If $V \in \mathfrak{U}$, then any bounded operator $A^{\prime}$ with $\exp \left(i A^{\prime}\right)=V$ is punctual, $r\left(A^{\prime}\right)=0$, and $\sigma\left(A^{\prime}\right) \subset(-\infty, \infty)$.

Proof. Let $\phi(\lambda)=\exp (i \lambda)$, and note that $\phi\left(\sigma\left(A^{\prime}\right)\right)=\sigma\left(\phi\left(A^{\prime}\right)\right)=\sigma(V) \subset \Gamma_{0}$ (from 6.2 and 5.3), so that $\sigma\left(A^{\prime}\right)$ is included in some open interval $J^{\prime} \subset(-\infty$, $\infty)$. Consider a point $\alpha_{0}^{\prime}$ and an interval $\jmath_{0}^{\prime}$, both arbitrary except for the requirement $\alpha_{0}^{\prime} \in J_{0}^{\prime} \subset J^{\prime}$; we can find a positive integer $m$ and a real number $\tau$ such that the function $g(\lambda)=\tau+\left(m^{-1}\right) \lambda$ satisfies $g\left(\Im_{0}^{\prime}\right) \ni \pi, g\left(\alpha_{0}^{\prime}\right) \in(0, \pi)$ and maps $J^{\prime}$ into an interval $J$ of diameter less than $\pi$.

Call $A=g\left(A^{\prime}\right)$. The function $\phi$ is one-to-one on $\Im \supset \sigma(A)$, and $\phi(A)=Y$ $=\exp \left\{i \tau I+i\left(m^{-1}\right) A\right\}$ is easily checked to be a member of $\mathfrak{u}$. By 5.3 we have $r(\phi(A))=0$; a repeated application of (6) now yields

$$
\phi\left\{g\left(r\left(A^{\prime}\right)\right)\right\}=\phi\left\{r\left(g\left(A^{\prime}\right)\right)\right\}=\phi\{r(A)\}=r\{\phi(A)\}=0,
$$

therefore $r\left(A^{\prime}\right)=0$.

It remains to show $\sigma_{0}\left(A^{\prime}\right) \subset p\left(A^{\prime}\right)$. To that effect, suppose $\alpha_{0}^{\prime} \in \sigma_{0}\left(A^{\prime}\right)$, in fact, let $\alpha_{0}^{\prime}$ be the only point of $\sigma\left(A^{\prime}\right)$ in $J_{0}^{\prime}$; if we call $\alpha=g\left(\alpha_{0}^{\prime}\right)$ and $(p, q)$ $=g\left(J_{0}^{\prime}\right)$, then $\alpha>0$ and

$$
\alpha \text { is the only point of } \sigma(A) \text { in }[p, \pi] \text {. }
$$

Hence $\lambda \in[p, \pi], \lambda \neq \alpha$, implies $\lambda \notin \sigma(A)$, which in turn necessitates $\lambda \in R(Y)$ (since $\phi(\lambda) \notin \sigma(Y)$ by (6), and 5.3(i)). Thus $(\alpha, \pi] \subset R(Y)$, and $\{Y\}_{\lambda}^{2}$ must 
be the same for any $\lambda$ in the interval of constancy $[p, \alpha)$; consequently $\{Y\}_{p}^{2}=\{Y\}_{\alpha-0}^{2}$. In view of 5.2 , our results can be gathered as follows:

$$
\{Y\}_{p}^{2}=\{Y\}_{\alpha-0}^{2}, \quad\{Y\}_{\alpha}^{2}=\{0\}=\{Y\}_{\pi}^{2} .
$$

The conclusion $\sigma_{0}\left(A^{\prime}\right) \subset p\left(A^{\prime}\right)$ is now at hand. We supposed $\alpha_{0}^{\prime} \in \sigma_{0}\left(A^{\prime}\right)$; if $\alpha_{0}^{\prime} \notin p\left(A^{\prime}\right)$, then (applying (6) twice) $\alpha \notin p(A), \phi(\alpha) \notin p(\phi(A))=p(Y)$, whence $\{Y\}_{\alpha-0}^{2}=\{Y\}_{\alpha}^{2}$ (by 5.3(ii)). This implies $\{Y\}_{p}^{2}=\{0\}=\{Y\}_{\pi}^{2}$ by (8), and $\{Y\}_{p}^{\alpha}=\{Y\}_{\pi}^{\alpha}(\kappa=1,2)$ by $4.3(\mathrm{c})$. But $\alpha \in[p, \pi]$, and 5.3(i) now shows that $\phi(\alpha) \notin \sigma(Y)$, which yields the contradiction $\alpha \notin \sigma(A)$ of (7).

6.4. Theorem V. If $\mathfrak{B}=\{B \mid \alpha B \in \mathfrak{R}(V), V \in \mathfrak{U}, \alpha$ complex $\}$, then any member of $\mathfrak{B} \cup \mathfrak{U}$ is punctual.

Proof. If $\alpha B \in \mathbb{R}(V), V \in \mathfrak{U}$, then $\exp (i\{-i \alpha B\})=V$, and 6.3 shows that $-i \alpha B$ is punctual; therefore $B$ is punctual (by (6)) whenever $B \in \mathfrak{B}$. If $Y \in \mathfrak{U}$ and $\lambda \in \sigma_{0}(Y)$, then $\mathfrak{l}_{\alpha} \subset \rho(Y)$ for some $\alpha$; we see from 3.8 that this implies the existence of a member $A$ of $\mathfrak{R}(Y)$ with $A=f(Y), f$ being one-to-one on $\mathfrak{b}=\sim \mathfrak{l}_{\alpha} \supset \sigma(Y)$. Hence $A \in \mathfrak{B}, A$ is punctual, and $Y$ is punctual by (6).

6.5. REMARK. If $B$ is bounded and self-adjoint, then $B \in \mathfrak{B}$; if $Y$ is unitary, then $Y \in \mathfrak{U}$ and $\mathfrak{R}(Y) \neq 0$ (see 9.1). It is apparent from 6.2 and the proofs of 6.3 and 6.4 that $r(X)=0$ when $X \in \mathfrak{B}$, or when $X \in \mathfrak{U}$ and $\mathfrak{R}(X) \neq 0$. We shall make no use of these properties. Examples of nonpunctual operators can be found in $[2$, p. 441$]$.

7. Two halving theorems.

7.1. Definition. $\|X\|=\sup \{\|X x\| \mid x \in \mathcal{D}(X),\|x\| \leqq 1\}$. The statement $\sum_{n=-\infty}^{\infty} \lambda_{n} X^{n}=Y$ will mean that

$$
\lim _{m, n \rightarrow \infty}\left\|\sum_{\nu=-m}^{n} \lambda_{\nu} X^{\nu}-Y\right\|=0, \quad \mathcal{D}(Y)=\mathscr{D}(X),
$$

and will carry the assumption that $\left\|X^{-1}\right\|<\infty, \mathcal{D}\left(X^{-1}\right)=\mathcal{D}(X)$ in case $\lambda_{n} \neq 0$ for some $n<0$.

7.2. Lemma. Let $\mathcal{N}$ be the null-space of $X$. The restriction of $Z$ to $\mathcal{N}$ is an operator $\Delta Z$ such that $\Phi(\Delta Z)=\mathcal{N}$ and $(\Delta Z) x=Z x$ for $x$ in $\mathcal{N}$. If $Z X=X Z$ (or if $Z<X$ ), then $\Delta Z$ maps $\mathcal{N}$ into itself, $\|\Delta Z\| \leqq\|Z\|, p(\Delta Z) \subset p(Z)$, and

$$
\begin{gathered}
\sum_{n=-\infty}^{\infty} \lambda_{n} Z^{n}=f(Z) \text { implies } f(\Delta Z)=\Delta f(Z), \\
r(\Delta Z)=0 \text { implies } \sigma(\Delta Z) \subset \sigma(Z) .
\end{gathered}
$$

The proof is omitted, (iv) is an immediate consequence of $[2$, Theorem 2.14.4].

7.3. Notation. In this section, $\kappa$ will be a fixed member of $\{1,2\}$. If $Z$ is an arbitrary operator, then $Z_{\mathrm{x}}$ and $\Delta Z$ will both denote the restriction of $Z$ to the null-space $\mathcal{N}$ of $[Y]^{\kappa}$. 
7.4. Theorem. Suppose $Y \in \mathfrak{u},-1 \notin p(Y)$, and $1 \notin p(Y)$. Then $Y<[Y]^{k}$, $Y_{k} \in \mathfrak{U}$, and

$$
\sigma\left(Y_{\kappa}\right) \subset \Gamma_{\kappa}=\left\{\zeta|| \zeta \mid=1, \quad(-1)^{\kappa} \operatorname{Im} \zeta \geqq 0\right\} .
$$

REMARK. The upper (lower) half of the unit-circumference contains thus $\sigma\left(Y_{\kappa}\right)$, depending on the choice of $\kappa$. From 7.1 and 4.2 we have $\mathcal{D}\left([Y]^{*}\right)$ $=\Phi(Y)$; hence

$$
\mathcal{N}=\left\{x \mid x \in \mathcal{D}(Y),[Y]^{k} x=0\right\} .
$$

Proof. From 4.2 and 2.1(c) follows $Y \prec[Y]^{\star}$. But $Y \in \mathfrak{U}$, and a repeated application of 7.2 therefore yields $\left\|\left(Y_{\kappa}\right)^{n}\right\|=\left\|(\Delta Y)^{n}\right\|=\left\|\Delta\left(Y^{n}\right)\right\| \leqq\left\|Y^{n}\right\| \leqq m_{0}$ $(n=0, \pm 1, \pm 2, \pm 3, \cdots)$. This shows that $Y_{k} \in \mathfrak{U}$, since $\mathcal{D}\left(Y_{k}\right)=\mathcal{N}$ is a reflexive space $[2$, Theorem 2.10 .3$]$. Let $\mathcal{N}_{\kappa}$ be the null-space of $\left[Y_{k}\right]^{\alpha}$; we now prove that $\mathcal{D}\left(Y_{k}\right)=\mathcal{N}_{k}$. It is enough to show that $\mathcal{N} \subset \mathcal{N}_{k}$, since we note (as in (1)) that $\mathcal{N}_{k}=\left\{x \mid x \in \mathcal{D}\left(Y_{k}\right),\left[Y_{k}\right]^{k} \cdot x=0\right\} \subset \mathcal{D}\left(Y_{k}\right)=\mathcal{N}$. Suppose $x \in \mathcal{N}$; if we write $f(Y)=[Y]^{k}$ and apply successively (1) and (iii), then

$$
0=f(Y) x=(\Delta f(Y)) x=f(\Delta Y) x=f\left(Y_{k}\right) x=\left[Y_{k}\right]^{\star} x,
$$

consequently $x \in \mathcal{N}_{k}$, and $\mathcal{N} \subset \mathcal{N}_{k}$. We have thus proved that $\mathcal{D}\left(Y_{k}\right)=\mathcal{N}_{k}$.

On the other hand, we see from $\pm 1 \notin p(Y) \supset p\left(Y_{k}\right)$ and 4.3(d) that $\mathcal{N}_{\kappa}=\left\{Y_{\kappa}\right\}_{0}^{\kappa}$; hence $\mathcal{D}\left(Y_{\kappa}\right)=\left\{Y_{\kappa}\right\}_{0}^{\kappa}$. In particular, $\mathcal{D}\left(Y_{2}\right)=\left\{Y_{2}\right\}_{0}^{2} \subset\left\{Y_{2}\right\}_{-\pi}^{2}$ $=\mathscr{D}\left(Y_{2}\right)$ (see 4.3); using 4.3(c), we obtain $\left\{Y_{2}\right\}_{-\pi}^{\kappa}=\left\{Y_{2}\right\}_{0}^{\kappa}$ for $\kappa=1$ and $\kappa=2$. In the notation of 5.1 , this means that $(-\pi, 0) \subset R\left(Y_{2}\right)$.

If $\zeta \in \sigma\left(Y_{2}\right)$, then $|\zeta|=1$ (since $\sigma\left(Y_{2}\right) \subset \Gamma_{0}$, by 5.3); writing now $\zeta=c^{i \lambda}$, $|\lambda| \leqq \pi$, we see that $\zeta \in \Gamma_{2}$, for otherwise $\lambda \in(-\pi, 0), \lambda \in R\left(Y_{2}\right)$, and $\zeta=\phi(\lambda)$ $\bigoplus \sigma\left(Y_{2}\right)$ by $5.3(\mathrm{i})$. This contradiction proves $\sigma\left(Y_{2}\right) \subset \Gamma_{2}$; an identical argument establishes $\sigma\left(Y_{1}\right) \subset \Gamma_{1}$.

7.5. THEOREM. Let $Y$ be as in 7.4, and suppose $A$ is a bounded operator with $\exp (i A)=Y$ and $\sigma(A) \subset[-\pi, \pi]$; then $\exp \left(i A_{\kappa}\right)=Y_{\kappa}$ and

$$
\sigma\left(A_{\kappa}\right) \subset J_{k} \text {, where } J_{1}=[-\pi, 0] \text { and } J_{2}=[0, \pi] \text {. }
$$

Proof. Set $\phi(\lambda)=e^{i \lambda}$. From $\phi(A)=Y$ and 2.1(c) we have $A<Y$; this implies $A<[Y]^{\kappa}$ (since $Y<[Y]^{\kappa}$ and 2.1). From (iii) follows $\phi\left(A_{\kappa}\right)=\Delta(\phi(A)$ ) $=\Delta Y=Y_{k}$. Note ${ }^{(13)}$ that $\sigma\left(A_{\kappa}\right) \subset \sigma(A) \subset[-\pi, \pi]$. In order to show that $\sigma\left(A_{\kappa}\right) \subset J_{\kappa}$, suppose $\lambda \in \sigma\left(A_{\kappa}\right)$. Then $|\lambda| \leqq \pi$ and $\left({ }^{10}\right) \phi(\lambda) \in \sigma\left(\phi\left(A_{\kappa}\right)\right)=\sigma\left(Y_{\kappa}\right)$, which implies, by 7.4 , that $(-1)^{\kappa} \operatorname{Im} \phi(\lambda)=(-1)^{\kappa} \operatorname{Im} e^{i \lambda} \geqq 0$; thus $(-1)^{\wedge} \lambda$ $\in(\{-\pi\} \cup[0, \pi])$. To complete the proof it now suffices to establish $\alpha_{\kappa}=-(-1)^{\kappa} \cdot \pi \notin \sigma\left(A_{\kappa}\right)$. Suppose otherwise; then $\alpha_{\kappa} \in \sigma_{0}\left(A_{\kappa}\right)$, and this necesitates $\left({ }^{13}\right)$ that $\alpha_{\kappa} \in p\left(A_{\kappa}\right)$. In view of 6.2 and 7.2 , we have $\phi\left(\alpha_{\kappa}\right) \in p\left(\phi\left(A_{\kappa}\right)\right)$ $=p\left(Y_{\kappa}\right) \subset p(Y)$, whence the contradiction $-1=\phi\left(\alpha_{\kappa}\right) \in p(Y)$.

${ }^{(13)}$ From $\phi\left(A_{\kappa}\right)=Y_{\kappa} \in \mathfrak{U}$ and 6.3 follows that $\sigma_{0}\left(A_{\kappa}\right) \subset p\left(A_{\kappa}\right)$ and $r\left(A_{\kappa}\right)=0$; therefore, $\sigma\left(A_{\kappa}\right) \subset \sigma(A)$ is a consequence of (iv). 
7.6. Lemma. Let $Y$ be as in 7.4 , and write $S=[Y]^{1}+[Y]^{2}$; then $Y \prec S$ and

$$
S X=S Z \text { implies } X=Z \text {. }
$$

Proof. That $Y<S$ follows from $Y<[Y]^{k}$ (see 7.4). In view of 4.2(2), we have $Y S=(2 i)^{-1}\left(Y^{2}-I\right)$; the hypothesis $S X=S Z$ implies therefore $(Y+I)$ $\cdot(Y-I) x_{0}=0, x_{0}=(X-Z) x$ for any $x$ in $\mathcal{D}(Y)$. It will be enough to show $x_{0}=0$; if $x_{0} \neq 0$, then $(Y \pm I) z=0$ for some $z \neq 0$, which contradicts the assumption $\pm 1 \notin p(Y)$.

\section{Uniqueness.}

8.1. TheOREM VI. If $Y \in \mathfrak{U}$ and neither 1 nor -1 belong to $p(Y)$, then there exists at most one bounded operator $A$ with $\exp (i A)=Y$ satisfying $\sigma(A)$ $C[-\pi, \pi]$. Moreover, $Y<A$ for such an $A$.

Proof. The notation will be the one specified in 7.3. Set $\kappa^{\prime} \neq \kappa, \kappa^{\prime}=1,2$ throughout; we first gather a few obvious facts (see 7.2).

$$
\begin{aligned}
Z_{\kappa} X & =Z X \quad \text { if } X x \in \mathcal{N} \text { when } x \in \mathcal{D}(X), \\
Z Y & =Y Z \text { implies } Z_{\kappa} Y_{\kappa}=Y_{\kappa} Z_{\kappa} \text { and } Z_{\kappa}[Y] \kappa^{\prime}=[Y]^{\prime} Z .
\end{aligned}
$$

To justify the last equality, we begin by observing that $Z[Y]^{\alpha^{\prime}}=[Y]^{\boldsymbol{k}^{\prime}} Z$, since $Y \prec[Y]^{\prime}$ (see 7.4 and 2.1). In view of (2), it suffices to note next that $y=[Y]^{\kappa^{\prime}} x \in \mathcal{N}$, since $[Y]^{\kappa^{\prime}} y=0$ (by $4.2(1)$ ).

We are now ready to prove the theorem. Set $\alpha=(-1)^{\star} \pi / 2$. From 7.4 we see that $-e^{i \alpha} \bigoplus \sigma\left(Y_{\kappa}\right)$; thus $\left\{p e^{i \alpha} \mid p \leqq 0\right\}=\mathfrak{l}_{\alpha} \subset \rho\left(Y_{\kappa}\right)$, and there exists, by 3.8, a unique $B^{(\kappa)}$ in $\mathfrak{l}\left(Y_{\kappa}\right)$ with $\sigma\left(B^{(\kappa)}\right) \subset \varepsilon_{\alpha}$. Note in passing that

$$
Y_{k} \prec B^{(\kappa)} \text {. }
$$

Let $A$ be a bounded operator with $\exp (i A)=Y$ and $\sigma(A) \subset[-\pi, \pi]$. From 7.5 we have $i A_{\kappa} \in \mathfrak{R}\left(Y_{\kappa}\right)$, and $\sigma\left(A_{\kappa}\right) \subset J_{\kappa}$; hence $\left({ }^{10}\right) \sigma\left(i A_{\kappa}\right) \subset z_{\alpha}$. Since $i A_{\kappa}$ thus shares these properties with $B^{(\kappa)}$, we must have $-i B^{(\kappa)}=A_{\kappa}$. But $\exp (i A)=Y$ implies $A Y=Y A$ (by 2.1); it therefore results from (3) that

$$
-\sum_{\kappa} i B^{(\kappa)}[Y]^{\kappa^{\prime}}=\sum_{\kappa} A_{\kappa}[Y] \kappa^{\prime}=\sum_{\kappa}[Y] \kappa^{\prime} A=S A .
$$

This holds for any bounded operator $A^{\prime}$ with $\exp \left(i A^{\prime}\right)=Y$ and $\sigma\left(A^{\prime}\right)$ $\subset[-\pi, \pi]$

$$
-\sum_{\kappa} i B^{(\kappa)}[Y]^{k^{\prime}}=S A=S A^{\prime} .
$$

The conclusion $A=A^{\prime}$ now follows from 7.6.

It only remains to prove $Y \prec A$. To that effect, suppose $Z Y=Y Z$; then $Z_{\kappa} B^{(\kappa)}=B^{(\kappa)} Z_{\kappa}$ (from (3) and (4)). This enables us to derive successively from (5), (3), (2), and (5)

$$
(S A) Z=-\sum_{\kappa} i B^{(\kappa)} Z_{\kappa}[Y] \kappa^{\prime}=-\sum_{\kappa} Z_{k} i B^{(\kappa)}[Y]^{\kappa^{\prime}}=Z(S A) .
$$


Now $Y<S$ (see 7.6) and therefore $Z S=S Z$, whence $Z(S A)=S(Z A)=S(A Z)$, which yields $Z A=A Z$ by 7.6 . This concludes the proof.

9. Conclusion. Let us suppose that $Y \in \mathfrak{U}$, and that neither 1 nor -1 are eigenvalues of $Y$. If a bounded operator $A$ can be found with $\exp (i A)=Y$ and $\sigma(A) \subset[-\pi, \pi]$, then it is (by Theorem VI) the only such operator, and $Y<A$. Using the notation defined in $\S 1$, we can then write $i A \in \mathbb{R}(Y), \sigma(i A)$ $C \mathrm{e}, \quad Y\left\langle i A\right.$; accordingly, $N_{\mathrm{e}}(Y)=1$ and $\log _{\mathrm{e}}(Y)=i A$. The existence of such an $A$ is guaranteed when $A$ is a unitary operator in Hilbert space (see 9.1). From 3.10 and 3.9 follows that any bounded operator $B$ with $\exp (i B)$ $=Y$ determines a finite family $\left\{P_{n} \mid n\right\}$ of idempotents such that $B=A$ $+2 \pi i N, N=\sum_{n} n P_{n}$; moreover, $B<Y<A<Y$.

9.1. THEOREM. If $Y$ is a unitary operator, then there exists a bounded operator $A$ with $\exp (i A)=Y$ and $\sigma(A) \subset[-\pi, \pi]$.

Proof. Since $-Y$ is also unitary, we can infer from Stone's Theorem $[9$, p. 307] the existence of an operator $B$ with $-Y=\exp (i 2 \pi B)$ and $\sigma(B)$ $C[0,1]$. Set $A=2 \pi B-\pi I$; then $-Y=-\exp (i A)$ and $\sigma(A) \subset[-\pi, \pi]$. This concludes the proof.

9.2. REMARK. Lemma 6.2 follows directly from certain results of Hille [2, p. 123 and p. 313]. The relevant part of this material (reproduced below) is unaltered by our removal of the additional condition $T \in \mathcal{D}(\Delta)$ found in $[2]\left({ }^{14}\right)$.

9.3. Definitions. We write $Z \in \mathfrak{B}_{1}$ whenever $Z x=0$ for some $x \neq 0$ in $\mathcal{D}(Z)$. The statement $Z \in \mathfrak{P}_{2}$ will indicate that the range of $Z$ is nondense in $\mathcal{D}(Z)$. Suppose $\kappa=1,2$ throughout. The following properties are immediately verified:

If $Z \in \mathfrak{B}_{k}$ and $A Z=Z A$, then $A Z \in \mathfrak{B}_{\kappa}$.

$$
\text { If } \prod_{n=1}^{m} Z_{n} \in \mathfrak{B}_{\kappa} \text {, then } Z_{n} \in \mathfrak{P}_{\kappa} \text { for some } n \leqq m \text {. }
$$

Finally, set $p_{\kappa}(Z)=\left\{\alpha \mid Z[\alpha] \in \mathfrak{B}_{\kappa}\right\}$, where $Z[\alpha]=\alpha I-Z$.

9.4. Lemma. Suppose $f$ is holomorphic on some open set $\mathfrak{b} \supset \sigma(X)$, so that $Y=f(X)$ can be defined as in 3.3. If $\alpha \in p_{k}(X)$, then $f(\alpha) \in p_{k}(Y)$.

Proof. Note that $\alpha \in \sigma(X)$. The function $g(\lambda)=[f(\alpha)-f(\lambda)] /(\alpha-\lambda)$ is holomorphic on $\mathfrak{b}$, and $(\alpha-\lambda) \cdot g(\lambda)=f(\alpha)-f(\lambda)$. Therefore

$$
X[\alpha] \cdot g(X)=Y[\beta]=g(X) \cdot X[\alpha], \quad \beta=f(\alpha) .
$$

But $X[\alpha] \in \mathfrak{B}_{k}$, and we conclude from (v) that $Y[\beta] \in \mathfrak{B}_{k}$.

${ }^{(14)}$ Hille defines $f(T)$ only when $T \in \mathfrak{D}(\Delta)$, in contrast to our broader definition, which is Dunford's $[1 ; 6]$. Hille proves that $f(T)$ is holomorphic on the connected subregion $\mathfrak{D}(\Delta)$ of $\mathcal{F}=\{T \mid f$ is holomorphic on $\sigma(T)\}$; it is easily inferred from his proof that $f(T)$ is locally holomorphic on $\mathcal{F}$. 
9.5. Lemma. The conditions of 9.4 imply that $p_{\kappa}(Y)=f\left(p_{k}(X)\right)$.

Proof. Suppose $\mu \in p_{x}(Y)$. By 9.4, it suffices to show that $\mu=f(\alpha)$ for some $\alpha \in p_{\mathfrak{k}}(X)$. We can find an open set $\mathfrak{a} \supset \sigma(X)$ with $\mathfrak{a} \subset \mathfrak{b}$, and such that $k(\lambda)=\mu$ $-f(\lambda)$ has only a finite number of zeros $z=\left\{\alpha_{n} \mid n\right\}$ in $\mathfrak{a}$, and none on $\partial \mathfrak{a}$. Note that $\xi \neq 0$ by the Dunford mapping theorem $\left({ }^{10}\right)$. If we set $\psi(\lambda)=\prod_{n}\left(\alpha_{n}-\lambda\right)$, then the function $h(\lambda)=\psi(\lambda) \cdot\{k(\lambda)\}^{-1}$ is holomorphic on $\mathfrak{a}$; the relation $(\mu-f(\lambda)) \cdot h(\lambda)=\psi(\lambda)$ implies therefore

$$
Y[\mu] \cdot h(X)=\prod_{n} X\left[\alpha_{n}\right]=h(X) \cdot Y[\mu] .
$$

But $Y[\mu] \in \mathfrak{B}_{\kappa}\left(\right.$ since $\left.\mu \in p_{k}(Y)\right)$, and $\prod_{n}\left(X\left[\alpha_{n}\right] \in \mathfrak{B}_{\kappa}\right.$ is now a consequence of (1) and (v). From (w) we accordingly infer that $X\left[\alpha_{n}\right] \in \mathfrak{P}_{\kappa}$ for some $n$, whence $\alpha_{n} \in p_{\kappa}(T)$. This concludes the proof, since $\mu=f\left(\alpha_{n}\right)$.

9.6. Proof of 6.2. Note that $p_{1}(Z)=p(Z)$, so that $f(\Lambda(X))=\Lambda(f(X))$ holds for $\Lambda=\sigma, p$ (by 9.5 and the Dunford mapping theorem $\left({ }^{10}\right)$ ). If $f$ is one-to-one on $\mathfrak{b}$, then $f(\Lambda(X))=\Lambda(f(X))$ implies

$$
\alpha \in \Lambda(X) \leftrightarrows f(\alpha) \in \Lambda(f(X))
$$

Consequently (u) holds for $\Lambda=\sigma, p_{1}, p_{2}$. That (u) holds also for $\Lambda=r$ is now seen to be a consequence of the definition $r(Z)=\left\{\alpha \mid \alpha \in p_{2}(Z)\right.$ and $\left.\alpha \notin p_{1}(Z)\right\}$.

\section{BIBLIOGRAPHY}

1. N. Dunford, Spectral theory. I. Convergence to projections, Trans. Amer. Math. Soc. vol. 54 (1943) pp. 185-217.

2. E. Hille, Functional analysis and semi-groups, Amer. Math. Soc. Colloquium Publications, vol. 31, New York, 1948.

3. E. R. Lorch, The integral representation of weakly almost-periodic transformations in reflexive vector spaces, Trans. Amer. Math. Soc. vol. 49 (1941) pp. 18-40.

4. - Proceedings of the symposium on spectral theory and differential problems, The Mathematics Department, Oklahoma Agric. and Mech. Coll., Stillwater, Oklahoma, 1951, pp. 263-264.

5. - The theory of analytic functions in normed abelian vector rings, Trans. Amer. Math. Soc. vol. 54 (1943) pp. 414-425.

6. F. Riesz and B. Sz.-Nagy, Lȩons d'analyse fonctionelle, Acad. Sci. Hongrie, Budapest, 1952.

7. A. E. Taylor, Spectral theory of closed distributive operators, Acta Math. vol. 84 (1951) pp. 189-223. 1952.

8. S. Saks and A. Zygmund, Analytic functions, Monografie Matematyczne, Warszawa,

9. M. H. Stone, Linear transformations in Hilbert space, Amer. Math. Soc. Colloquium Publications, vol. 15, New York, 1932.

10. B. Sz.-Nagy, On uniformly bounded linear transformations in Hilbert space, Acta Univ. Szeged vol. 11 (1947) pp. 151-157.

UNIVERSITY of CALIFoRNia, Berkeley, Calif.

Purdue University, LafayetTe, IND. 